Pacific Journal of Mathematic 


\title{
ON THE BOUNDARY CONTINUITY OF CONFORMAL MAPS
}

\author{
Ch. Pommerenke
}

Let the function $f$ map the unit disk $D$ conformally onto the domain $G$ in $\hat{\mathbf{C}}=\mathbf{C} \cup\{\infty\}$. The prime end theory of Carathéodory gives a completely geometric characterization of the boundary behavior of $f$. Prime ends are defined in terms of crosscuts of $G$.

Our aim is to give a geometric description of the boundary behavior of $f$ that refers only to the boundary $\partial G$ and not to the domain itself. It can therefore be applied to any complementary domain of a connected closed set in $\hat{\mathbf{C}}$. Our description will however be incomplete because we will have to allow exceptional sets.

1. Introduction and results. We say that $f$ has the angular limit $f(\zeta)$ at $\zeta \in \partial \mathbf{D}$ if

$$
f(\zeta)=\lim _{z \rightarrow \zeta, z \in \Delta} f(z) \in \hat{\mathbf{C}}
$$

exists for every Stolz angle $\Delta$ at $\zeta$; we shall always denote by $f(\zeta)$ the angular limit if it exists. A theorem of Beurling [1] (see e.g. [4, p. 56] [8, p. $341,344])$ states that the angular limit $f(\zeta)$ exists for $\zeta \in B$ where $\operatorname{cap}(\partial \mathbf{D} \backslash B)=0$ and furthermore that

$$
\operatorname{cap}\{\zeta \in B: f(\zeta)=\omega\}=0 \quad \text { for } \omega \in \hat{\mathbf{C}}
$$

here cap denotes the logarithmic capacity.

We shall say that $f$ is continuous at $\zeta \in \partial \mathbf{D}$ if $f$ has a continuous extension to $\mathbf{D} \cup\{\zeta\}$, that is, if $f(z) \rightarrow f(\zeta)$ as $z \rightarrow \zeta, z \in \mathbf{D}$. Our first result states that discontinuity tends to imply injectivity.

THEOREM 1. Let $f$ map D conformally onto $G$. Then there is a partition

$$
\partial \mathbf{D}=A_{0} \cup A_{1} \cup A_{2}
$$

such that

(i) $\operatorname{cap} A_{0}=0$,

(ii) the angular limit $f(\zeta)$ exists for every $\zeta \in A_{1}$, and $f$ is one-to-one on $A_{1}$,

(iii) $f$ is continuous at each $\zeta \in A_{2}$, and $f$ is exactly two-to-one on $A_{2}$. 
Let $E$ be a continuum in $\hat{\mathbf{C}}$. The point $\omega \in E$ will be called accessible if there exists a Jordan arc $C$ with endpoint $\omega$ such that $C \cap E=\{\omega\}$. If $G$ is a component of $\hat{\mathbf{C}} \backslash E$ we say that $\omega$ is accessible from $G$ if there is a Jordan arc $C$ ending at $\omega$ such that $C \subset G \cup\{\omega\}$; every accessible point is accessible from some component. If $f$ maps $\mathbf{D}$ conformally onto the component $G$ of $\hat{\mathbf{C}} \backslash E$, then every angular limit $f(\zeta)$ is accessible from $G$. Conversely, if $\omega \in E$ is accessible from $G$ then there is at least one $\zeta \in \partial \mathbf{D}$ such that $\omega=f(\zeta)[8$, p. 277].

We have to introduce another topological concept. We call $\omega \in E$ a quasi-isolated accessible point if there is a neighborhood $V$ of $\omega$ such that $\omega$ is the only accessible point in the component of $E \cap \bar{V}$ containing $\omega$. Thus the other accessible points of $E$ cannot be connected to $\omega$ by a subcontinuum of small diameter.

As an example, we consider first the classical unsymmetric comb

$$
E_{1}=[-1+i, 1+i] \cup[0, i] \cup \bigcup_{n=1}^{\infty}\left[\frac{1}{n}, \frac{1}{n}+i\right] .
$$

The point 0 is not quasi-isolated because, for $0<r<1$, its component of $E \cap\{|z| \leq r\}$ is $[0$, ir $]$ and all points on this segment are accessible. Consider now the symmetric comb

$$
E_{2}=E_{1} \cup \bigcup_{n=1}^{\infty}\left[-\frac{1}{n},-\frac{1}{n}+i\right] .
$$

Then 0 is accessible but quasi-isolated because now no point of $(0, i r)$ is accessible.

Our next result is essentially topological.

THEOREM 2. Let $f$ map $\mathbf{D}$ conformally onto $G$. Then, for all $\zeta \in \partial \mathbf{D}$ with at most countably many exceptions, the function $f$ is continuous at $\zeta$, if and only if

(i) the angular limit $f(\zeta)$ exists, and

(ii) the accessible point $f(\zeta)$ of $\partial G$ is not quasi-isolated.

The classical comb shows that there may be exceptional values of $\zeta$, and indeed our ideas about the boundary behavior of conformal maps seem to be strongly influenced by the exceptional cases.

COROllary 1. Let $f$ map D conformally onto $G$. Then there is a partition

$$
\partial \mathbf{D}=B_{0} \cup B_{1} \cup B_{2}
$$


such that

(i) $\operatorname{cap} B_{0}=0$,

(ii) $f(\zeta)$ exists for $\zeta \in B_{1}$ and $f(\zeta)$ is a quasi-isolated accessible point of $\partial G$

(iii) $f$ is continuous at each $\zeta \in B_{2}$.

This is a consequence of Theorem 2 because, for conformal maps, the set of $\zeta \in \partial \mathbf{D}$ where $f(\zeta)$ does not exist has zero capacity, by Beurling's theorem. The corollary is not true for arbitrary topological mappings; it is easy to construct a topological self-mapping of $\mathbf{D}$ that is nowhere continuous on $\partial \mathbf{D}$.

Corollary 2. Let $f$ be a conformal mapping of $\mathbf{D}$ onto $G$. If all points of $\partial G$ are accessible then $f$ is continuous on $\partial \mathbf{D}$ except possibly for a set of zero capacity.

It is well-known that $f$ is continuous in $\overline{\mathbf{D}}$ if every boundary point is accessible from $G$ "from all sides." We have made a weaker assumption but then we have to allow for exceptions. If $E_{1}$ is again the classical comb defined by (1.2) then every boundary point of $G=\hat{\mathbf{C}} \backslash E_{1}$ is accessible but $f$ is not continuous in $\overline{\mathbf{D}}$.

This corollary follows from Corollary 1 because there are no quasiisolated points if every point of $\partial G$ is accessible.

Corollary 3 (B. Rodin). Let $f$ map D conformally onto $G$ and suppose that

$$
g(f(z))=f\left(e^{2 \pi i \alpha} z\right), \quad \alpha \in \mathbf{R} \backslash \mathbf{Q},
$$

where $g$ is continuous in $\bar{G}$. If all points of $\partial G$ are accessible from $G$, then $\partial G$ is a Jordan curve in $\hat{\mathbf{C}}$.

This result is of interest for Siegel disks in the theory of iterations. It is due to Rodin [9, Theorem 3]. The only new aspect is that his additional hypothesis that $f$ is continuous for at least one $\zeta \in \partial \mathbf{D}$ follows automatically, by Corollary 2, from his assumption that each point of $\partial G$ is accessible from $G$. Moeckel [6] has given an example of a function $f$ satisfying (1.5) with a function $g$ continuous in $\bar{G}$ such that every point of $\partial G$ is accessible (though not always from $G$ ) and $f$ has countably many discontinuities on $\partial \mathbf{D}$.

I want to thank Professor Burt Rodin for our discussions. His result was the starting point of the present investigation. 
2. Proof of Theorem 1. The proofs are based on two remarkable topological countability theorems. A triod is the union of three Jordan arcs that begin at a common point but are otherwise disjoint. The following result is due to R. L. Moore [7].

MoORe Triod TheOREM. Every disjoint collection of triods in the plane is countable.

Let $f$ be any function defined in $\mathbf{D}$. For $\zeta \in \partial \mathbf{D}$, the left-hand cluster set $C_{L}(\zeta)$ is defined by

$$
\begin{aligned}
& C_{L}(\zeta)=\left\{w \in \hat{\mathbf{C}} \text { there are } z_{n} \in \mathbf{D}\right. \text { with } \\
& \left.\qquad z_{n} \rightarrow \zeta, \arg z_{n} \geq \arg \zeta, f\left(z_{n}\right) \rightarrow w\right\} .
\end{aligned}
$$

The right-hand cluster set $C_{R}(\zeta)$ is defined similarly with $\arg z_{n} \leq \arg \zeta$ instead, and $C(\zeta)=C_{L}(\zeta) \cup C_{R}(\zeta)$ is the unrestricted cluster set. Note that $f$ is continuous at $\zeta$ if and only if $C(\zeta)$ is a singleton. The following result is due to Collingwood [3] [4, p. 83].

Collingwood Symmetry Theorem. Let $f$ be defined in $\mathbf{D}$. Then

$$
C_{L}(\zeta)=C_{R}(\zeta)=C(\zeta)
$$

for all $\zeta \in \partial \mathbf{D}$ with at most countably many exceptions.

The point $\omega \in E$ is called a cut point of the continuum $E$ if $E \backslash\{\omega\}$ is not connected. It follows from the plane separation theorem [10, p. 34] that $\omega \in E$ is a cut point of $E$ if and only if there is a Jordan curve $J \subset \hat{\mathbf{C}}$ with $J \cap E=\{\omega\}$ that separates $E \backslash\{\omega\}$. If $E$ bounds a domain $G$ then $J \backslash\{\omega\}$ has to lie in $G$.

Proof of Theorem 1. Let $A_{0}^{\prime}$ denote the set of $\zeta \in \partial \mathbf{D}$ for which the angular limit $f(\zeta)$ does not exist. Beurling's theorem states that cap $A_{0}^{\prime}=0$. Furthermore, let

$$
A_{2}^{\prime}=\left\{\zeta \in \partial \mathbf{D} \backslash A_{0}^{\prime}: f(\zeta) \text { is a cut point of } \partial G\right\}
$$

and let $A_{1}=\partial \mathbf{D} \backslash\left(A_{0}^{\prime} \cup A_{2}^{\prime}\right)$.

We show first that (ii) holds. Suppose that $f$ is not one-to-one on $A_{1}$. Then there exist $\zeta, \zeta^{*} \in A_{1}$ such that $f(\zeta)=f\left(\zeta^{*}\right)=\omega$. Then

$$
J=f(\zeta S) \cup f(\zeta * S), \quad S \equiv[0,1]
$$


is a Jordan curve that intersects $\partial G$ only at $\omega$. By Beurling's theorem, $f$ has angular limits different from $\omega$ on both arcs of $\partial \mathbf{D} \backslash\left\{\zeta, \zeta^{*}\right\}$. Hence we conclude that there are points of $\partial G$ in both components of $\hat{\mathbf{C}} \backslash J$. Therefore $\omega$ is a cut point of $\partial G$, contrary to our assumption $\zeta \in A_{1} \subset$ $\partial \mathbf{D} \backslash A_{2}^{\prime}$.

Let now $\zeta \in A_{2}^{\prime}$. Then $\omega=f(\zeta)$ is a cut point of $\partial G$ by (2.2), and there is a Jordan curve $J \subset G \cup\{\omega\}$ through $\omega$ that separates $\partial G \backslash\{\omega\}$. The open Jordan arc $Q=f^{-1}(J \backslash\{\omega\}) \subset \mathbf{D}$ ends at definite points $\zeta_{1}, \zeta_{2} \in \partial \mathbf{D}[8$, p. 267].

If $\zeta_{1}=\zeta_{2}$ then $Q \cup\left\{\zeta_{1}\right\}$ is a Jordan curve. Its inner domain $H$ lies in D, and $f(z) \rightarrow \omega$ as $z \rightarrow \zeta, z \in H$ by a theorem of Lehto and Virtanen [5]. Hence $f(H)$ is one of the components of $\hat{\mathbf{C}} \backslash J$. Since $f(H) \subset G$ and since $J$ is to separate $\partial G \backslash\{\omega\}$, we conclude that the case $\zeta_{1}=\zeta_{2}$ is impossible. Since $f$ has the angular limit $\omega$ at $\zeta_{1}$ and at $\zeta_{2}[8$, p. 268] we thus see that there is at least one $\zeta^{*} \neq \zeta$ with $f\left(\zeta^{*}\right)=\omega$.

Let $E_{0}$ be the set of $\omega \in \partial G$ for which there are at least three points $\zeta_{1}$ with angular limits $f\left(\zeta_{j}\right)=\omega$. For $\omega \in E_{0}$,

$$
f\left(\zeta_{1} S_{0}\right) \cup f\left(\zeta_{2} S_{0}\right) \cup f\left(\zeta_{3} S_{0}\right), \quad S_{0} \equiv[1 / 2,1],
$$

is a triod because $f$ is univalent in $\mathbf{D}$. If $\omega^{*} \in E_{0}, \omega^{*} \neq \omega$, then the corresponding triods are disjoint. Hence it follows from the Moore triod theorem that $E_{0}$ is countable. Hence

$$
A_{0}^{\prime \prime}=\left\{\zeta \in \partial \mathbf{D} \backslash A_{0}^{\prime}: f(\zeta) \in E_{0}\right\}
$$

has zero capacity by Beurling's theorem. If $\zeta \in A_{2}^{\prime} \backslash A_{0}^{\prime \prime}$ then there is exactly one further $\zeta^{*} \in A_{2}^{\prime} \backslash A_{0}^{\prime \prime}$ such that $f\left(\zeta^{*}\right)=f(\zeta)$.

Finally we define $A_{0}$ as $A_{0}^{\prime} \cup A_{0}^{\prime \prime}$ together with all points $\zeta \in A_{2} \backslash A_{0}^{\prime \prime}$ such that either $C_{L}(\zeta) \neq C_{R}(\zeta)$ or $C_{L}\left(\zeta^{*}\right) \neq C_{R}\left(\zeta^{*}\right)$; by the Collingwood symmetry theorem, there are at most countably many such points. Hence cap $A_{0}=0$ so that (i) holds. We define $A_{2}=A_{2}^{\prime} \backslash A_{0}$. Then we have the partition $\partial \mathbf{D}=A_{0} \cup A_{1} \cup A_{2}$, and $f$ is exactly two-to-one on $A_{2}$.

In order to establish (iii) we have to show that $C(\zeta)$ is a singleton for each $\zeta \in A_{2}$. Let $\zeta^{*}$ be the other point in $A_{2}$ with $f\left(\zeta^{*}\right)=f(\zeta)$ and consider the Jordan curve $J$ defined by (2.3). Let $H_{L}, H_{R}$ be the components of $\hat{\mathbf{C}} \backslash J$; we may assume that the points to the left of $f(\zeta S)$ lie in $H_{L}$. Then those to the right of $f(\zeta S)$ lie in $H_{R}$. Hence

$$
C_{L}(\zeta) \subset \bar{H}_{L}, \quad C_{R}(\zeta) \subset \bar{H}_{R} .
$$

Since $C_{L}(\zeta)=C_{R}(\zeta)=C(\zeta)$ because of $\zeta \notin A_{0}$, we conclude that

$$
C(\zeta) \subset \bar{H}_{L} \cap \bar{H}_{R}=J
$$

and since $C(\zeta) \subset \partial G$ and $J \cap \partial G=\{f(\zeta)\}$ it follows that $C(\zeta)=\{f(\zeta)\}$, and this completes the proof of Theorem 1 . 
3. Proof of Theorem 2. (a) Let first $f$ be continuous at $\zeta \in \partial \mathbf{D}$. It is clear that the angular limit $f(\zeta)$ exists. Let $V$ be a neighborhood of $f(\zeta)$. Then there is a disk around $\zeta$ such that its intersection $U$ with $\mathbf{D}$ satisfies $f(U) \subset V$. Hence

$$
F \equiv \overline{f(U)} \cap \partial G \subset \bar{U} \cap \partial G .
$$

Since $F$ is connected it follows that $F$ lies in the component of $\bar{V} \cap \partial G$ that contains $f(\zeta)$.

Since $f$ is conformal there exists $\zeta^{\prime} \in \partial U \cap \partial \mathbf{D}$ such that the angular limit $f\left(\zeta^{\prime}\right)$ exists and is different from $f(\zeta)$, for instance by Beurling's theorem quoted above. Hence $f\left(\zeta^{\prime}\right)$ is also an accessible point in $F$. It follows that $f(\zeta)$ is not quasi-isolated.

(b) In order to prove the converse direction we may assume that $\infty \in G$ so that $\partial G$ lies in $\mathbf{C}$. We shall not use that $f$ is meromorphic so that $f$ may be any topological mapping from $\mathbf{D}$ onto $G$.

Let $A$ denote the set of all $\zeta \in \partial \mathbf{D}$ such that the angular limit $f(\zeta)$ exists and $f(\zeta)$ is not quasi-isolated. Let $G_{k}$ denote the components of $\hat{\mathbf{C}} \backslash \partial G$. For $\zeta \in A$ and $n \in \mathbf{N}$, let $E_{n}(\zeta)$ denote the component of

$$
\{w:|w-f(\zeta)| \leq 1 / n\} \cap \partial G
$$

that contains $f(\zeta)$. Since $\omega$ is not quasi-isolated there is an accessible point $\omega_{n}(\zeta) \in E_{n}(\zeta)$ with $\omega_{n}(\zeta) \neq f(\zeta)$.

Let $A_{n k}$ denote the set of $\zeta \in A$ such that $\omega_{n}(\zeta)$ is accessible from the component $G_{k}$; these sets need not be disjoint. Let

$$
X=\left\{\zeta \in A: C_{L}(\zeta) \neq C_{R}(\zeta)\right\} \cup \bigcup_{A_{n k} \text { singleton }} A_{n k} .
$$

The first set is countable by the Collingwood symmetry theorem. Hence $X$ is countable.

Let now $\zeta \in A \backslash X$. We shall show that $f$ is continuous at $\zeta$. Let $\Gamma=\{f(r \zeta): 1 / 2 \leq r \leq 1\}$. We have $\zeta \in A_{n k}$ for some $k=k(n)$ and there is a Jordan arc $\Gamma_{n} \subset G_{k} \cup\left\{\omega_{n}(\zeta)\right\}$ that ends at $\omega_{n}(\zeta)$. We distinguish two cases:

Case 1. Let first $\omega_{n}(\zeta)$ be accessible from $G$. Let $P_{n} \subset G$ be a Jordan arc connecting the other endpoints of $\Gamma_{n}$ and $\Gamma$ (without otherwise meeting $\Gamma_{n}$ and $\Gamma$ ) and let

$$
L_{n}=\Gamma \cup P_{n} \cup \Gamma_{n} \cup E_{n}(\zeta) .
$$


Since $\Gamma \cup P_{n} \cup \Gamma_{n}$ is a crosscut of $\hat{\mathbf{C}} \backslash E_{n}(\zeta)$ and since $E_{n}(\zeta)$ is a continuum, the points lying locally on the two sides of $\Gamma$ belong to different components of $\hat{\mathbf{C}} \backslash L_{n}$, say $H_{n}$ and $H_{n}^{*}$.

Case II. Let now $\omega_{n}(\zeta)$ be accessible from some component $G_{k} \neq G$. Since $\zeta \notin X$ we see from (3.1) that there exists $\zeta_{n}^{\prime} \in A_{n k}$ with $\zeta_{n}^{\prime} \neq \zeta_{n}$. Hence there are Jordan arcs $\Gamma_{n}$ and $\Gamma_{n}^{\prime}$ that lie in $G_{k}$ except for their endpoints $\omega_{n}(\zeta)$ and $\omega_{n}\left(\zeta^{\prime}\right)$. Let $P_{n}$ be a Jordan arc in $G_{k}$ that connects the other endpoints of $\Gamma_{n}$ and $\Gamma_{n}^{\prime}$. Furthermore let $Q_{n}$ be a Jordan arc in $G$ from $f(\zeta / 2)$ to $f\left(\zeta_{n}^{\prime} / 2\right)$. We set $\Gamma_{n}^{*}=\left\{f\left(r \zeta_{n}^{\prime}\right): 1 / 2 \leq r \leq 1\right\}$ and

$$
L_{n}=\left(\Gamma_{n} \cup P_{n} \cup \Gamma_{n}^{\prime}\right) \cup\left(\Gamma \cup Q_{n} \cup \Gamma_{n}^{*}\right) \cup E_{n}(\zeta) \cup E_{n}\left(\zeta^{\prime}\right)
$$

Since $E_{n}(\zeta)$ and $E_{n}\left(\zeta_{n}^{\prime}\right)$ are continua and since $\Gamma_{n} \cup P_{n} \cup \Gamma_{n}^{\prime}$ and $\Gamma \cup Q_{n}$ $\cup \Gamma_{n}^{*}$ are disjoint Jordan arcs connecting $E_{n}(\zeta)$ with $E_{n}\left(\zeta^{\prime}\right)$, the points lying locally on the two sides of $\Gamma$ lie in different components of $\mathbf{C} \backslash L_{n}$, say $H_{n}$ and $H_{n}^{*}$.

Now we consider both cases together. Let $j>1$, let $U_{J}$ and $U_{j}^{*}$ be the "left" and "right" components of $\{z \in \mathbf{D}:|z-\zeta|<1 / j\} \backslash[\zeta / 2, \zeta]$. Then $f\left(U_{j}\right)$ intersects $H_{n}$ and $f\left(U_{j}^{*}\right)$ intersects $H_{n}^{*}$ if we label the components $H_{n}$ and $H_{n}^{*}$ of $\mathbf{C} \backslash L_{n}$ accordingly. If $j$ is large then $f\left(U_{j}\right)$ and $f\left(U_{j}^{*}\right)$ do not intersect $L_{n}$ as we see from (3.2) or (3.3) because $f$ is a homeomorphism from $\mathbf{D}$ onto $G$. Hence there exists $j_{n}$ such that

$$
f\left(U_{j_{n}}\right) \subset H_{n}, \quad f\left(U_{j_{n}}^{*}\right) \subset H_{n}^{*} .
$$

It follows from (2.1) and the corresponding definition of $C_{R}(\zeta)$ and from (3.4) that, for $n=1,2, \ldots$,

$$
C_{L}(\zeta) \subset \overline{f\left(U_{j_{n}}\right)} \subset \bar{H}_{n}, \quad C_{R}(\zeta) \subset \overline{f\left(U_{J_{n}}^{*}\right)} \subset \bar{H}_{n}^{*} .
$$

Since $\zeta \notin X$ we therefore obtain from (3.1) that

$$
C(\zeta)=C_{L}(\zeta) \cap C_{R}(\zeta) \subset \bar{H}_{n} \cap \bar{H}_{n}^{*} \subset L_{n} .
$$

Furthermore $C(\zeta) \subset \partial G$. Hence we conclude

$$
C(\zeta) \subset L_{n} \cap \partial G \subset E_{n}(\zeta) \text { or } \subset E_{n}(\zeta) \cup E_{n}\left(\zeta_{n}^{\prime}\right)
$$

from (3.2) for Case I and from (3.3) for Case II, respectively.

In Case I, we immediately get from (3.5) that

$$
\operatorname{diam} C(\zeta) \leq \operatorname{diam} E_{n}(\zeta) \leq 2 / n ;
$$


this inequality holds also in Case II if $E_{n}(\zeta)$ and $E_{n}\left(\zeta_{n}^{\prime}\right)$ are disjoint because then (3.5) implies that the continuum $C(\zeta)$ lies in $E_{n}(\zeta)$. If $E_{n}(\zeta)$ and $E_{n}\left(\zeta_{n}^{\prime}\right)$ intersect then we obtain from (3.5) that

$$
\operatorname{diam} C(\zeta) \leq \operatorname{diam}\left[E_{n}(\zeta) \cup E_{n}\left(\zeta_{n}^{\prime}\right)\right] \leq 4 / n .
$$

Since $C(\zeta)$ is independent of $n$ we conclude in all cases that $C(\zeta)$ is a singleton so that $f$ is continuous at $\zeta$.

\section{REFERENCES}

[1] A. Beurling, Ensembles exceptionnels, Acta Math., 72 (1940), 1-13.

[2] C. Carathéodory, Über die Begrenzung einfach zusammenhängender Gebiete, Math. Ann., 73 (1913), 323-370.

[3] E. F. Collingwood, Cluster sets of arbitrary functions, Proc. Nat. Acad. Sci. U.S.A., 46 (1960), 1236-1242.

[5] O. Lehto and K. I. Virtanen, Boundary behavior and normal meromorphic functions, Acta Math., 97 (1957), 47-65.

[6] R. Moeckel, Rotations of the closures of some simply connected domains, preprint Univ. of Minnesota, 1984.

[7] R. L. Moore, Concerning triods in the plane and the junction points of plane continua, Proc. Nat. Acad. Sci. U.S.A., 14 (1928), 85-88.

[8] Ch. Pommerenke, Univalent Functions, Vandenhoeck \& Ruprecht, Göttingen 1975.

[9] B. Rodin, Intrinsic rotations of simply connected regions, Complex Variables Theory Appl., 2 (1984), 319-326.

[10] G. T. Whyburn, Topological Analysis, Princeton Univ. Press 1958.

Received July 6, 1984. This research was in part supported by the National Science Foundation.

University of California, SAN Diego

LA Jolla, CA 92093

AND

TECHNISCHE UNIVERSITÄT

D-1000 BERLIN 12

W. GERMANY 


\section{PACIFIC JOURNAL OF MATHEMATICS EDITORS}

\author{
V. S. VARADARAJAN (Managing Editor) \\ University of California \\ Los Angeles, CA 90024 \\ Charles R. DePrima \\ California Institute of Technology \\ Pasadena, CA 91125 \\ R. FINN \\ Stanford University \\ Stanford, CA 94305
}

\author{
HeRmanN FlaschKa \\ University of Arizona \\ Tucson, AZ 857.21
}

RAMESH A. GANGOLli

University of Washington

Seattle, WA 98195

ROBION KIRBY

University of California

Berkeley, CA 94720
C. C. MOORE

University of California

Berkeley, CA 94720

H. SAMELSON

Stanford University

Stanford, CA 94305

HAROLD STARK

University of California, San Diego

La Jolla, CA 92093

\section{ASSOCIATE EDITORS}
R. ARENS
E. F. BECKENBACH
B. H. NeumanN
F. WOLF
K. YoSHIDA
(1906-1982)

\section{SUPPORTING INSTITUTIONS}

\begin{abstract}
UNIVERSITY OF ARIZONA
UNIVERSITY OF BRITISH COLUMBIA

CALIFORNIA INSTITUTE OF TECHNOLOGY

UNIVERSITY OF CALIFORNIA

MONTANA STATE UNIVERSITY

UNIVERSITY OF NEVADA, RENO

NEW MEXICO STATE UNIVERSITY

OREGON STATE UNIVERSITY
\end{abstract}

\author{
UNIVERSITY OF OREGON \\ UNIVERSITY OF SOUTHERN CALIFORNIA \\ STANFORD UNIVERSITY \\ UNIVERSITY OF HAWAII \\ UNIVERSITY OF TOKYO \\ UNIVERSITY OF UTAH \\ WASHINGTON STATE UNIVERSITY \\ UNIVERSITY OF WASHINGTON
}

The Supporting Institutions listed above contribute to the cost of publication of this Journal, but they are not owners or publishers and have no responsibility for its content or policies.

Mathematical papers intended for publication in the Pacific Journal of Mathematics should be in typed form or offset-reproduced (not dittoed), double spaced with large margins. Please do not use built up fractions in the text of the manuscript. However, you may use them in the displayed equations. Underline Greek letters in red, German in green, and script in blue. The first paragraph must be capable of being used separately as a synopsis of the entire paper. In particular it should contain no bibliographic references. Please propose a heading for the odd numbered pages of less than 35 characters. Manuscripts, in triplicate, may be sent to any one of the editors. Please classify according to the scheme of Math. Reviews, Index to Vol. 39. Supply name and address of author to whom proofs should be sent. All other communications should be addressed to the managing editor, or Elaine Barth, University of California, Los Angeles, California 90024.

There are page-charges associated with articles appearing in the Pacific Journal of Mathematics. These charges are expected to be paid by the author's University, Government Agency or Company. If the author or authors do not have access to such Institutional support these charges are waived. Single authors will receive 50 free reprints; joint authors will receive a total of 100 free reprints. Additional copies may be obtained at cost in multiples of 50 .

The Pacific Journal of Mathematics is issued monthly as of January 1966. Regular subscription rate: $\$ 190.00$ a year (5 Vols., 10 issues). Special rate: $\$ 66.00$ a year to individual members of supporting institutions.

Subscriptions, orders for numbers issued in the last three calendar years, and changes of address should be sent to Pacific Journal of Mathematics, P.O. Box 969, Carmel Valley, CA 93924, U.S.A. Old back numbers obtainable from Kraus Periodicals Co., Route 100, Millwood, NY 10546.

The Pacific Journal of Mathematics at P.O. Box 969, Carmel Valley, CA 93924 (ISSN 0030-8730) publishes 5 volumes per year. Application to mail at Second-class postage rates is pending at Carmel Valley, California, and additional mailing offices. Postmaster: Send address changes to Pacific Journal of Mathematics, P.O. Box 969, Carmel Valley, CA 93924.

PUBLISHED BY PACIFIC JOURNAL OF MATHEMATICS, A NON-PROFIT CORPORATION

Copyright $\odot 1985$ by Pacific Journal of Mathematics 


\section{Pacific Journal of Mathematics}

Vol. 120, No. $2 \quad$ October, 1985

Philip Marshall Anselone and Mike Treuden, Regular operator

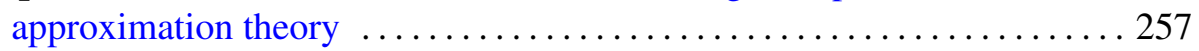

Giuseppe Baccella, Semiprime $\aleph-Q F 3$ rings $\ldots \ldots \ldots \ldots \ldots \ldots \ldots \ldots . \ldots \ldots$

Earl Robert Berkson and Thomas Alastair Gillespie, The generalized M.

Riesz theorem and transference $\ldots \ldots \ldots \ldots \ldots \ldots \ldots \ldots . \ldots 279$

Joachim Boidol, A Galois-correspondence for general locally compact

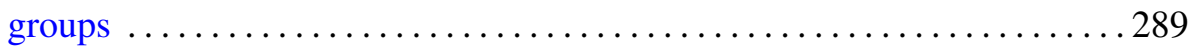

Joseph Eugene D'Atri, Josef Dorfmeister and Yan Da Zhao, The isotropy

representation for homogeneous Siegel domains ............... 295

C. Debiève, On Banach spaces having a Radon-Nikodým dual

Michael Aaron Freedman, Existence of strong solutions to singular

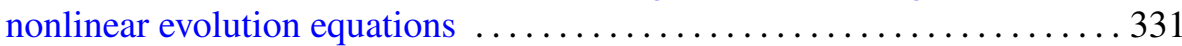

Francisco Jose Freniche, Grothendieck locally convex spaces of continuous

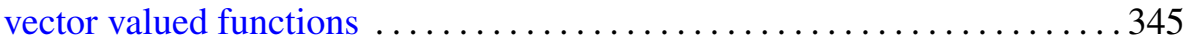

Hans-Peter Künzi and Peter Fletcher, Extension properties induced by complete quasi-uniformities . ............................ 357

Takaŝi Kusano, Charles Andrew Swanson and Hiroyuki Usami, Pairs of

positive solutions of quasilinear elliptic equations in exterior domains . . 385

Angel Rafael Larotonda and Ignacio Zalduendo, Spectral sets as Banach

manifolds

J. Martínez-Maurica and C. Pérez García, A new approach to the

Kreı̆n-Milman theorem

Christian Pommerenke, On the boundary continuity of conformal maps . . . 423

M. V. Subba Rao, Some Rogers-Ramanujan type partition theorems

Stephen Edwin Wilson, Bicontactual regular maps .........

Jaap C. S. P. van der Woude, Characterizations of (H)PI extensions

Kichoon Yang, Deformation of submanifolds of real projective space

Subhashis Nag, Errata: "On the holomorphy of maps from a complex to a

real manifold" 